Bull. Austral. Math. Soc.

54A05, 06А99, 06B99, 54C60

VoL. 40 (1989) [129-145]

\title{
CONVERGENCE IN NEIGHBOURHOOD LATTICES
}

\author{
Frank P. Prokop
}

\begin{abstract}
A consideration of the separation properties of pre-neighbourhood lattices, leads to the definition and characterisation of $T_{1}$-neighbourhood lattices in terms of the properties of the neighbourhood mapping, independently of points. It is then shown that if net convergence is defined in neighbourhood lattices as a consequence of replacing 'point' by 'set' in topological convergence, then the limits of convergent nets are unique. The relationship between continuity and convergence is established with the proof of the statement that a residuated function between conditionally complete $T_{1}$-neighbourhood lattices is continuous if and only if it preserves the limit of convergent nets. If $\mathcal{P}(X)$ denotes the power set of $X$, then the observation that a filter in a topological space $(X, T)$ is a net in $\mathcal{P}(X)$ leads to a discussion of the net convergence of a filter as a special case of net convergence. Particular attention is paid to maximal filters, Fréchet filters and to the filter generated by the limit elements of a net. Further, if the 'filter' convergence of a filter $F$ in a topological space $(X, T)$ is given by $F \stackrel{f}{\rightarrow} x$, if $\eta(x) \subseteq F$, then the relationship between 'filter' convergence and the net convergence of a filter in $\mathcal{P}(X)$ is established. Finally, it is proved that, in the neighbourhood system 'lifted' from a topological space to $\mathcal{P}(\mathcal{P}(X))$, the continuous image of a filter which converges to a singleton set is a convergent filter with the appropriate image set as the linit.
\end{abstract}

\section{INTRODUCTION}

Neighbourhood lattices were shown in [20] to have the appropriate structure for generalising the non-convergence aspects of topological spaces. This paper will show that not only can convergence be defined in neighbourhood lattices, but also that limits of convergent nets are unique and that a residuated function between conditionally complete $T_{1}$-neighbourhood lattices is continuous if and only if it preserves the limit of convergent nets. Further, we will show that in conditionally complete $T_{1}$-lattices there is a unified theory of convergence, which encompasses both nets and filters.

We will make use of the following notation and conventions:

If $L$ is an orthocomplemented lattice, then ' will be used to denote complementation; $I$ will be used to denote an arbitrary indexing set; if $P$ is a poset, $\vee$ and $\wedge$ will represent the operations of sup and inf; while $U$ and $\cap$ will be used to denote whe set theoretic operations of union and intersection, $\bigvee_{\alpha \in 1} x_{\alpha}$ will be used to represent. $\bigvee\left\{x_{\alpha} \mid \alpha \in 1\right\}$, with similar abbreviations used for $\bigwedge_{\alpha \in I} x_{\alpha}, \bigcup_{\alpha \in 1} A_{\alpha}$, and $\bigcap_{\alpha \in I} A_{\alpha} ; 1$ will represent the greatest element of $P$ and $\emptyset$ will represent the least element of $P$;

Received 20 October 1988

Copyright Clearance Centre, Inc. Serial-fee code: 0004-9729/89 $\$$ A2.00+0.00. 
$\mathcal{F}(P)=\{F \mid F$ is a filter of $P\} ; \mathcal{I}(P)=\{I \mid I$ is an ideal of $P\}$; if $x \in P$, then $[x)=\{y \mid y \in P \& y \geqslant x\}$ and $(x]=\{y \mid y \in P \& y \leqslant x\}$. In addition, the following definitions, which are discussed fully in [20], will be used. If $P$ is an $\wedge$-semi-lattice, then a function $\eta: P \rightarrow \mathcal{F}(P)$ is a filter mapping. If $\eta$ is a filter mapping on $P$, then $g \in P$ is neighbourhood open (or simply open) if $\eta(g)=[g)$. Further, if $\eta$ is a filter mapping on $P$, we will let $G=\{g \mid g \in P \& \eta(g)=[g)\}$. A filter mapping $\eta$ is a pre-neighbourhood mapping if $(\forall x, t \in P)(t \in \eta(x) \Leftrightarrow(\exists g \in G)(x \leqslant g \leqslant t))$. A filter mapping $\eta$ is a neighbourhood mapping if i) $G$ is a $V$-semi-complete sublattice of $L$, and ii) $\emptyset \in L \Rightarrow \eta(\emptyset)=[\emptyset)$. If $(X, T)$ is a topological space, then $\eta: \mathcal{P}(X) \rightarrow \mathcal{F}(\mathcal{P}(X))$ defined by $\eta(A)=\{N:(\exists g \in T)(A \subseteq g \subseteq N)\}$ is the induced neighbourhood mapping on $\mathcal{P}(X)$ and the pair $(\mathcal{P}(X), \eta)$ is the induced neighbourhood lattice of $(X, T)$. Finally, if $X$ and $Y$ are sets and $f: X \rightarrow Y$ is a function, then the direct image function, $f_{*}: \mathcal{P}(X) \rightarrow \mathcal{P}(Y)$ is given by $f_{*}(A)=\{f(a) \mid a \in A\}$, and the inverse image function, $f^{*}: \mathcal{P}(Y) \rightarrow \mathcal{P}(X)$ is given by $f^{*}(B)=\{x \mid x \in X \& f(x) \in B\}$.

The proofs of those Lemmas which are straight forward computations and which follow directly from the corresponding definitions will be omitted.

\section{2. $T_{0}$ AND $T_{1}$ NEIGHBOURHOOD LATTICES}

We have shown in [20] the specific way in which the neighbourhoods of a set in a topological space are determined by the neighbourhoods of its points. This leads to the question: "To what extent are the separation properties 'inherited' by the induced neighbourhood mapping?"

We will begin this section by noting that a topological space $(X, T)$ is a $T_{0}$-space if and only if the induced neighbourhood mapping, $\eta$, restricted to the points of $\mathcal{P}(X)$, is one to one. Hence, being a $T_{0}$-space is properly a property of 'points'. Thus, we have

DEFINITION 1: An atomic pre-neighbourhood lattice $(L, \eta)$ is a $T_{0}$-lattice if $\eta$, restricted to the atoms of $L$, is one to one.

We will now examine how $T_{1}$-topological spaces can be characterised in terms of the properties of the induced neighbourhood mapping $\eta$. We begin by looking at the proof of the standard topological statement that a topological space is $T_{1}$ if and only if the neighbourhood mapping is one to one.

Result 2. Let $(X, T)$ be a topological space, and $(\mathcal{P}(X), \eta)$ be the induced neighbourhood lattice. Then $(X, T)$ is a $T_{1}$-space $\Leftrightarrow \eta$ is a one to one function.

Proof: Let $(X, T)$ be a $T_{1}$-space and let $A, B \in \mathcal{P}(X)$. Then $A \neq B \Rightarrow$ $(\exists a \in A)(a \notin B) \Rightarrow\{a\}^{\prime} \notin \eta(A) \Rightarrow \eta(A) \neq \eta(B)$. Conversely, suppose $\eta$ is $1-1$ on $\mathcal{P}(X)$, and $x \in X .\{x\}^{\prime} \neq X \Rightarrow \eta\left(\{x\}^{\prime}\right) \neq \eta(X)$. Thus, $\eta\left(\{x\}^{\prime}\right)=\left[\{x\}^{\prime}\right)$ and $\{x\}^{\prime}$ is open. 
It is clear that a $T_{0}$-space is not a $T_{1}$-space if and only if there exists $A, B \in \mathcal{P}(X)$, $A \neq B$, for which $\eta(A)=\eta(B)$. Further, being a $T_{1}$-space is not a point dependent property. Finally, the proof of Result 2 uses only the pre-neighbourhood properties of $\eta$.

Thus, we can define a $T_{1}$-pre-neighbourhood lattice.

Definition 3: A pre-neighbourhood lattice $(L, \eta)$ is a $T_{1}$-lattice if $\eta: L \rightarrow \mathcal{F}(L)$ is a one to one function.

We will now give an alternative characterisation of $T_{1}$-lattices.

TheOREM 4. Let $(L, \eta)$ be a conditionally complete pre-neighbourhood lattice. Then $(L, \eta)$ is a $T_{1}$-lattice $\Leftrightarrow(\forall a \in L)\left(\bigwedge_{x \in \eta(a)} x=a\right)$.

Proof: $x \in \eta(a) \Rightarrow a \leqslant x$. Thus, $\bigwedge_{x \in \eta(a)} x$ exists in $L$ and $\bigwedge_{x \in \eta(a)} x \geqslant a$. Let $L$ be a $T_{1}$-lattice, and let $b=\bigwedge_{x \in \eta(a)} x$. If $b>a$, then $\eta(a) \supset \eta(b)$ (proper). But, $g$ an open element and $g \in \eta(a) \Rightarrow b \leqslant g \Rightarrow g \in \eta(b)$. A contradiction. Conversely, $a \neq b$ and $\eta(a)=\eta(b) \Rightarrow \bigwedge_{x \in \eta(a)} x=\bigwedge_{y \in \eta(b)} y \Rightarrow a=b$. A contradiction.

It follows from Theorem 4 that $(L, \eta)$ is a $T_{1}$-lattice $\Leftrightarrow(\forall a \in L)\left(\bigwedge_{g \in \eta(a) \cap G} g=a\right)$.

We can now state as a corollary to Theorem 4, a standard equivalent condition for a topological space to be a $T_{1}$-space. The "usual" topological proof of this result uses complementation and the fact that points are closed in a $T_{1}$-space. We have shown that the proof depends only on the conditional completeness of $\mathcal{P}(X)$, and on the pre-neighbourhood and one to one properties of $\eta$.

COROLlaRY 4.1. Let $(X, T)$ be a topological space, and let $(\mathcal{P}(X), \eta)$ be the induced neighbourhood lattice. Then $(X, T)$ is a $T_{1}$-space $\Leftrightarrow(\forall A \in \mathcal{P}(X))$ $\left(A=\bigcap_{y \in \eta(A)} y=\bigcap \eta(A)\right)$.

Proof: $(X, T)$ is a $T_{1}$-space $\Leftrightarrow(\mathcal{P}(X), \eta)$ is a $T_{1}$-lattice.

We will now show that in a $T_{1}$-lattice the order relation on pre-neighbourhood images must reflect the order relation in the lattice.

THEOREM 5. Let $(L, \eta)$ be a conditionally complete pre-neighbourhood lattice. Then $(L, \eta)$ is a $T_{1}$-lattice $\Leftrightarrow(\forall a, b \in L)(\eta(a) \subseteq \eta(b) \Rightarrow b \leqslant a)$.

Proof: Let $(L, \eta)$ be a $T_{1}$-lattice, and $\eta(a) \subseteq \eta(b) . x \in \eta(a) \Rightarrow(\exists g \in \eta(a) \cap G)$ 
( $a \leqslant g \leqslant x$ ). But, $g \in \eta(b) \Rightarrow b \leqslant x$. Thus, $b \leqslant \bigwedge_{x \in \eta(a)} x=a$. Conversely, $a \neq b$ and $\eta(a)=\eta(b) \Rightarrow a=b$. A contradiction.

It should be noted that a finite $T_{1}$-lattice must have the discrete pre-neighbourhood system. Thus, being a $T_{1}$-lattice is an 'infinitary' property.

We will now show that $T_{1}$-topological spaces are 'self-dual' in the sense that both the induced neighbourhood mapping and the induced dual neighbourhood mappings are one to one.

TheOREM 6. Let $(X, T)$ be a topological space and let $\gamma$ be the induced dual neighbourhood mapping. Then $(X, T)$ is a $T_{1}$-space $\Leftrightarrow \gamma: \mathcal{P}(X) \rightarrow \mathcal{I}(\mathcal{P}(X))$ is a one to one function.

Proof: Let $\gamma$ be one to one. Since $\gamma(\emptyset)=\{\emptyset\}, \gamma(\{x\}) \neq\{\emptyset\}$. Thus, $\gamma(\{x\})=(x]$ and $\{x\}$ is closed. Conversely, let $(X, T)$ be a $T_{1}$-space. $A \neq B \Rightarrow(\exists b \in B)(b \notin A)$. Thus, $\{b\} \in \gamma(B)$ and $\{b\} \notin \gamma(A)$. Hence, $\gamma(A) \neq \gamma(B)$.

We will conclude this section by noting that if $L$ is an atomistic lattice and $L$ has either a Čech closure operator or a closure operator defined, then $L$ is a $T_{1}$-lattice if and only if 'points' are closed. More precisely, it follows from Theorem 18 in [20], that if $L$ is a complete atomistic lattice, ${ }^{c}: L \rightarrow L$ is a Čech closure mapping, $\mathcal{A}=$ $\{a \mid a$ is an atom of $L\}$ and if $\gamma$ is the dual neighbourhood mapping determined by $H=\left\{h: h=h^{c}\right\}$, then $(L, \gamma)$ is a $T_{1}$-lattice if and only if $(\forall a \in \mathcal{A})\left(a^{c}=a^{-}=a\right)$, that is, if and only if points are closed. Further, we can state that if $(L, ')$ is an orthocomplemented atomistic neighbourhood lattice and $x \in L$ is defined to be closed if $x^{\prime}$ is open, then $(L, \eta)$ is a $T_{1}$-lattice if and only if $(\forall a \in \mathcal{A})(a$ is closed).

\section{Convergence of Nets in Neighbourhood Lattices}

We have shown in [20] that in all statements about continuous functions which were not concerned with convergence, points could be replaced by sets and neighbourhoods of points by neighbourhoods of sets. If we attempt to continue this substitution of points by sets in developing a theory of convergence, we are faced with the classical question "How does one define the convergence of a net or a sequence of sets?" The classical $\lim$ inf and $\lim$ sup of a sequence of sets was an attempt to answer this question so that the answer 'made sense' for nested sequences of sets, without regard to topological considerations. Our identification of $x$ with $\{x\}$ demands that a theory of convergence in neighbourhood lattices must begin by 'making sense' when the sets which make up a net of sets are each singleton sets, that is, we want $x_{n} \rightarrow x_{0}$ to be identified with $\left\{x_{n}\right\} \rightarrow\left\{x_{0}\right\}$. 
Further, we will show that in neighbourhood lattices as in topological spaces, convergence can be approached using either nets or filters and the theories that are generated are equivalent.

Finally, it should be noted that as the generality of the theory increases, we now allow sets as well as points to converge, the requirement for unique limits decreases. For limits of convergent nets to be unique in a topological space, the space must be Hausdorff. However, in pre-neighbourhood lattices limits of convergent nets are always unique. To 'see' how this can happen, consider the sequence $\left\{A_{n}\right\}$ of sets in $P$ with the usual topology, defined by

$$
(\forall n \in \mathrm{N})\left(A_{n}=\left\{\begin{array}{ll}
\{1\} & \text { if } n \text { is even } \\
\{-1\} & \text { if } n \text { is odd }
\end{array}\right) .\right.
$$

This sequence of sets corresponds to the sequence $\left\{a_{n}\right\}$ of elements of $\mathbf{R}$ given by

$$
(\forall n \in N)\left(a_{n}=\left\{\begin{array}{ll}
1 & \text { if } n \text { is even } \\
-1 & \text { if } n \text { is odd }
\end{array}\right) .\right.
$$

The sequence $\left\{a_{n}\right\}$ has no limit in $R$, but we will show that the sequence $\left\{\mathcal{A}_{n}\right\}$ converges to $\{1,-1\}$, that is, the sequence of singleton sets converges to the set of accumulation points (or cluster points) of the corresponding sequence of points, and this set of accumulation points is unique.

We will now develop a theory of convergence in neighbourhood lattices based on nets.

DEFINITION 7: Let $(L, \eta)$ be a pre-neighbourhood lattice, let $D$ be a directed set, and let $\left\{x_{d} \mid d \in D\right\}$ be a net in $L . x \in L$ is a limit element of the net $\left\{x_{d}\right\}$ if $(\forall y \in \eta(x))(\exists q \in D)\left((\forall p \in D)\left(p \geqslant q \Rightarrow x_{p} \leqslant y\right)\right)$.

It is clear that if $1 \in L$, then 1 is a limit element of every net in $L$. Further, if $\left\{x_{n} \mid n \in N\right\}$ is a sequence of points in a topological space, then the set of linit elements of the sequence includes the 'usual' set of limit points of the sequence.

LEMMA 8. Let $(L, \eta)$ be a pre-neighbourhood lattice, and let $\left\{x_{d} \mid d \in D\right\}$ be a net in $L$, and $\Lambda=\left\{z \mid z\right.$ is an limit element of $\left.\left\{x_{d}\right\}\right\}$. Then

(i) $x \in \Lambda \& z \geqslant x \Rightarrow z \in \Lambda$.

(ii) $x \in \Lambda \Leftrightarrow(\forall g \in \eta(x) \cap G)(\exists q \in D)\left((\forall p \in D)\left(p \geqslant q \Rightarrow x_{p} \leqslant g\right)\right)$.

Definition 9: Let $(L, \eta)$ be a pre-neighbourhood lattice, $\left\{x_{d} \mid d \in D\right\}$ be a net in $L$, and $\Lambda=\left\{z \mid z\right.$ is an limit element of $\left.\left\{x_{d}\right\}\right\}$. We will say that $\left\{x_{d}\right\}$ converges to $x$ (written $\left.x_{d} \rightarrow x\right) \Leftrightarrow($ i) $x \in \Lambda$, and (ii) $(\forall a \in \Lambda)(x \leqslant a)$.

In Theorem 10, we will show that a net converges if and only if the set of limit elements of the net is a principal filter. 
Theorem 10. Let $(L, \eta)$ be a pre-neighbourhood lattice, $\left\{x_{d} \mid d \in D\right\}$ be a net in $L$, and let $\Lambda=\left\{x \mid z\right.$ is a limit element of $\left.\left\{x_{d}\right\}\right\}$. Then $x_{d} \rightarrow x \Leftrightarrow \Lambda=[x)$.

Proof: Suppose $x_{d} \rightarrow x$. From Lemma 8 and Definition $\theta$ (ii), $\Lambda$ is a filter. $\Lambda=[x$ ) by Definition 9 (i). The converse is immediate from Definition $\theta$.

Corollary 10.1. Let $(L, \eta)$ be a pre-neighbourhood lattice, $\left\{x_{d} \mid d \in D\right\}$ be a net in $L$, and $\Lambda=\left\{z \mid z\right.$ is an limit element of $\left.\left\{x_{d}\right\}\right\}$. Then $x_{d} \rightarrow x \Leftrightarrow x \in \Lambda$, and $x=\bigwedge_{x \in \Lambda} z$

It follows from Theorem 10 , that if $(L, \eta)$ is a pre-neighbourhood lattice with $\emptyset$, then $x_{d} \rightarrow \emptyset \Leftrightarrow \Lambda=[\emptyset) \Leftrightarrow(\exists q \in D)\left((\forall p \in D)\left(p \geqslant q \Rightarrow x_{p}=\emptyset\right)\right)$.

We will now show that limits of convergent nets are unique. This is not as surprising as it might first appear, since when a net of points in a topological space converges, it converges to a set which is uniquely determined by the accumulation elements of the net.

Theorem 11. Let $(L, \eta)$ be a pre-neighbourhood lattice, $\left\{x_{d} \mid d \in D\right\}$ be a net in $L$, and $\Lambda=\left\{z \mid z\right.$ is an limit element of $\left.\left\{x_{d}\right\}\right\}$. If $x_{d} \rightarrow x$ and $x_{d} \rightarrow y$, then $x=y$.

PRoOF: $\Lambda=[x)=[y) \Rightarrow x=y$.

Definition 9 agrees with the usual definition of net convergence in a topological space when limits are unique. More specifically, let $\left\{x_{d} \mid d \in D\right\}$ be a net in $(X, T)$, $\left\{\left\{x_{d}\right\} \mid d \in D\right\}$ be the corresponding net in $(\mathcal{P}(X), \eta), \Lambda_{x_{d}}$ be the set of limit elements of $\left\{x_{d}\right\}$, and $\Lambda_{\left\{x_{d}\right\}}$ be the set of limit elements of $\left\{\left\{x_{d}\right\}\right\}$. Now, $x \in \Lambda_{x_{d}} \Rightarrow$ $(\forall y \in \eta(x))(\exists q \in D)\left(p \geqslant q \Rightarrow x_{p} \in y\right) \Rightarrow(\forall y \in \eta(\{x\}))(\exists q \in D)\left(p \geqslant q \Rightarrow\left\{x_{p}\right\} \subseteq y\right) \Rightarrow$ $\{x\} \in \Lambda_{\left\{x_{d}\right\}}$. However, if $\left\{x_{d}\right\}$ converges to two different points, $\left\{\left\{x_{d}\right\}\right\}$ does not converge, since $\Lambda_{\left\{x_{d}\right\}}$ cannot be a proper principal filter. To take into account nonuniqueness of limits, we could define topological convergence in $\mathcal{P}(X)$ as follows: If $(X, T)$ is a topological space and $(\mathcal{P}(X), \eta)$ is induced neighbourhood lattice, then a net $\left\{x_{d} \mid d \in D\right\}$ in $X$ converges topologically to a point $x$ if and only if $\{x\} \in \Lambda_{\left\{x_{d}\right\}}$. Clearly, a net $\left\{x_{d} \mid d \in D\right\}$ in a Hausdorff space $(X, T)$ converges topologically to $x$ if and only if $\left\{x_{d}\right\} \rightarrow\{x\}$ in $(\mathcal{P}(X), \eta)$, that is, if and only if $\Lambda_{\left\{x_{d}\right\}}=[\{x\})$, or equivalently if and only if $\Lambda_{\left\{x_{d}\right\}}$ is a maximal filter generated by a singleton.

The following examples indicate how Corollary 10.1 can be used to establish the convergence or non-convergence of nets. We will let $R$ be the real numbers with the usual topology and $(\mathcal{P}(R), \eta)$ be the induced neighbourhood lattice of the usual topology on $R$. If we define $(\forall n \in N)\left(a_{n}=1 / n\right)$ then $\Lambda=[\{0\})$, and $\{0\}=\bigcap_{z \in \Lambda} z$. Thus $a_{n} \rightarrow 0$. If we define $(\forall n \in \mathbf{N})\left(b_{n}=n\right)$ and $(\forall k \in \mathbf{N})\left(w_{k}=\mathbf{N}-\{1,2, \ldots k\}\right)$, then $(\forall k \in N)\left(w_{k} \in \Lambda\right)$. Hence, $\bigcap_{z \in \Lambda} z \subseteq \bigcap_{k \in N} w_{k}=\emptyset$. Thus $\left\{b_{n}\right\}$ does not converge. Sim- 
ilarly, if we let $\left\{x_{n}\right\}$ be the sequence of rational numbers, then $\forall x \in R,\{x\}^{\prime} \in \Lambda$. Hence, $\bigcap_{z \in \Lambda} z \subseteq \bigcap_{x \in \mathbb{R}}\{x\}^{\prime}=\emptyset$. Thus, $\left\{x_{n}\right\}$ does not converge. If we let $A_{n}=(0,1 / n)$, then $\{0\}$, any superset of $\{0\}$, and $(0,1)$ are limit elements of $\left\{A_{n} \mid n \in N\right\}$. Thus, since $\Lambda$ cannot be a proper principal filter, $\left\{A_{n} \mid b \in N\right\}$ does not converge. Hence, a nested decreasing sequence of sets need not have a limit.

We will summarise the convergence aspects of non-increasing and non-decreasing sequences of sets in a $T_{1}$-topological space in Theorem 12.

Theorem 12. Let $(X, T)$ be a $T_{1}$-space, and let $(\mathcal{P}(X), \eta)$, be the induced neighbourhood lattice.

(i) If $A_{1} \subseteq A_{2} \subseteq A_{3} \subseteq \ldots \subseteq A_{n} \subseteq \ldots$ is a nested non-decreasing sequence of sets in $\mathcal{P}(X)$, then $A_{n} \rightarrow \bigcup_{i=1}^{\infty} A_{i}$.

(ii) If $A_{1} \supseteq A_{2} \supseteq A_{3} \supseteq \ldots \supseteq A_{n} \supseteq \ldots$ is a nested non-increasing sequence of sets in $\mathcal{P}(X)$, and $A=\bigcap_{i=1}^{\infty} A_{i}$, then $A_{n} \rightarrow A \Leftrightarrow(\forall g \in \eta(A) \cap G)(\exists q \in N)$ $\&\left(A_{q} \subseteq q\right)$.

Proof: (i) We will let $x=\bigcup_{i=1}^{\infty} A_{i}$, and show that $\Lambda=[x) . \quad t \in \Lambda \Rightarrow$ $(\forall y \in \eta(t))(\exists q \in \mathrm{N})\left(p \geqslant q \Rightarrow A_{p} \subseteq y\right) \Rightarrow(\forall y \in \eta(t))\left(x \subseteq y \Rightarrow x \subseteq \bigcap_{x \in \eta(t)} z=t\right) \Rightarrow t \in$ [x). Conversely, $t \in[x) \& g \in \eta(t) \cap G \Rightarrow \bigcup_{i=1}^{\infty} A_{i} \subseteq g \Rightarrow t \in \Lambda$.

(ii) By Theorem 10, $A_{n} \rightarrow A \Rightarrow(\forall g \in \eta(A) \cap G)(\exists q \in N)\left(A_{q} \subseteq g\right)$. Conversely, $(\forall g \in \eta(A) \cap G)(\exists q \in N)\left(A_{q} \subseteq g\right) \Rightarrow(\forall g \in \eta(A) \cap G)(A \subseteq g) \Rightarrow A \in \Lambda \Rightarrow[A) \subseteq \Lambda$. But, $x \in \Lambda \Rightarrow(\forall g \in \eta(x) \cap G)(\exists q \in N)(p \geqslant q) \Rightarrow A_{p} \subseteq g \Rightarrow(\forall g \in \eta(x) \cap G)(A \subseteq g) \Rightarrow$ $\eta(x) \subseteq \eta(A) \Rightarrow A \subseteq x \Rightarrow \Lambda \subseteq[A)$.

In particular, Theorem 12(i) proves that for a nested non-decreasing sequence of sets in the induced neighbourhood lattice of the usual topology on $R$, the sequence converges to the classical limit set defined in terms of lim inf and lim sup. Further, Theorem 12(ii) gives a necessary and sufficient condition for a nested non-increasing sequence of sets in the induced neighbourhood lattice of the usual topology on $\mathbf{R}$, to converge to the classical limit. For example, if we define $(\forall n \in N)\left(A_{n}=[-1 / n,(1+n) / n]\right)$, then Theorem 12(ii) is satisfied by $\left\{A_{n}\right\}$ and $A_{n} \rightarrow[0,1]$.

We will make use of Lemma 13 in proving that continuous residuated mappings preserve the linits of convergent nets in conditionally complete $T_{1}$-lattices.

LEMMA 13. Let $\left(L_{1}, \eta_{1}\right)$ and $\left(L_{2}, \eta_{2}\right)$ be pre-neighbourhood lattices with $G_{1}$ and $G_{2}$ the respective sets of open elements, let $f: L_{1} \rightarrow L_{2}$ be a continuous residuated 
mapping, $\left\{x_{d} \mid d \in D\right\}$ be a net in $L_{1}, \Lambda=\left\{z \mid z\right.$ is an limit element of $\left.\left\{x_{d}\right\}\right\}$, and $\Lambda_{f}=\left\{z \mid z\right.$ is a limit element of $\left\{f\left(x_{d}\right)\right\}$. Then:

(i) $\eta_{2}(y) \subseteq \eta_{2}(f(x)) \Leftrightarrow\left(\forall g \in G_{2}\right)\left(g \in \eta_{2}(y) \Rightarrow f^{+}(g) \in \eta_{1}(x)\right)$,

(ii) $x_{d} \rightarrow x \Rightarrow f(x) \in \Lambda_{f}$;

(iii) if $x_{d} \rightarrow x$ and $f\left(x_{d}\right) \rightarrow y$, then

(a) $y \leqslant f(x)$, (b) $g \in \eta_{2}(y) \cap G_{2} \Rightarrow f^{+}(g) \in \Lambda$, (c) $\eta_{2}(y)=\eta_{2}(f(x))$, and (d) $y \in G_{2} \Rightarrow y=f(x)$.

Proof: (i) Suppose $g \in G_{2} \cap \eta_{2}(y) \Rightarrow f^{+}(g) \in \eta_{1}(x)$. Now $t \in \eta_{2}(y) \Rightarrow$ $\left(\exists g \in G_{2} \cap \eta_{2}(y)\right)(y \leqslant g \leqslant t)$. But, $f^{+}(g) \in \eta_{1}(x) \Rightarrow x \leqslant f^{+}(g) \Rightarrow f(x) \leqslant f\left(f^{+}(g)\right) \leqslant$ $g \leqslant t$. Thus, $t \in \eta_{2}(f(x))$. Suppose $\eta_{2}(y) \subseteq \eta_{2}(f(x))$. Then $g \in G_{2} \cap \eta_{2}(f(x)) \Rightarrow x \leqslant$ $f^{+}(f(x)) \leqslant f^{+}(g)$. But, $f^{+}(g) \in G_{1} \Rightarrow f^{+}(g) \in \eta_{1}(x)$.

(ii) $x_{d} \rightarrow x \Rightarrow \Lambda=[x) . g \in \eta_{2}(f(x)) \cap G_{2} \Rightarrow f(x) \leqslant g \Rightarrow x \leqslant f^{+}(f(x)) \leqslant f^{+}(g)$. But the continuity of $f$ and the convergence of $\left\{x_{d}\right\} \Rightarrow(\exists q \in D)\left(p \geqslant q \Rightarrow x_{p} \leqslant f^{+}(g)\right)$. Hence, if $p \geqslant q, f\left(x_{p}\right) \leqslant g$, and $f(x) \in \Lambda_{f}$.

(iii) This follows from (ii) and the definition of continuity.

Example 14 will show that even if $\left(L_{1}, \eta_{1}\right)$ and $\left(L_{2}, \eta_{2}\right)$ are distributive neighbourhood lattices, and $f$ is a continuous residuated mapping, then $x_{d} \rightarrow x$ and $f\left(x_{d}\right) \rightarrow y$ need not imply $y=f(x)$.

Example 14. Let $L_{1}=\{1, a, b, c, \emptyset\}, L_{2}=\left\{1^{\prime}, \alpha, \beta, \emptyset^{\prime}\right\}$ be chains, $\left(L_{1}, \eta_{1}\right)$ and $\left(L_{2}, \eta_{2}\right)$ be neighbourhood lattices with $G_{1}=\{1, a, b, c, \emptyset\}$ and $G_{2}=\left\{1^{\prime}, \beta, \emptyset^{\prime}\right\}$ the respective sets of open elements.

If $f: L_{1} \rightarrow L_{2}$ is given by $f(1)=1^{\prime}, f(c)=\beta=f(b), f(a)=\alpha$, and $f(\emptyset)=f\left(\emptyset^{\prime}\right)$, then $f$ is a residuated lattice homomorphism of $L_{1}$ onto $L_{2}$ with the residual mapping $f^{+}: L_{2} \rightarrow L_{1}$ given by $f^{+}\left(1^{\prime}\right)=1, f^{+}(\beta)=c, f^{+}(\alpha)=a$, and $f^{+}\left(\emptyset^{\prime}\right)=\emptyset$. Further, since $\left(\forall g \in G_{2}\right)\left(f^{+}(g) \in G_{1}\right), f$ is continuous. Now, if $\left\{x_{n}\right\}$ is the sequence given by $(\forall n \in N)\left(x_{n}=b\right)$, then, since $\Lambda=\{b, c, 1\}=[b), x_{n} \rightarrow b$. However, $\left\{f\left(x_{n}\right)\right\}=\{\beta\}$, and $\Lambda_{f}=\left\{\alpha, \beta, 1^{\prime}\right\}=[\alpha)$. Thus, $f\left(x_{n}\right) \rightarrow \alpha \neq \beta=f(b)$. It should be noted that $L_{2}$ is not a $T_{1}$-lattice, and that $f^{+}$is not continuous at $\alpha$.

Theorem 15 proves that a residuated function between conditionally complete $T_{1}$ neighbourhood lattices is continuous if and only if it preserves the limit of convergent nets. The proof of Theorem 15 makes use of Lemma 18, which is proved in Paragraph 4.

Theorem 15. Let $\left(L_{1}, \eta_{1}\right)$ and $\left(L_{2}, \eta_{2}\right)$ be conditionally complete $T_{1}$-lattices, $f: L_{1} \rightarrow L_{2}$ be a residuated mapping, $\left\{x_{d} \mid d \in D\right\}$ be a net in $L_{1}, \Lambda=\{z \mid$ $z$ is a limit element of $\left.\left\{x_{d}\right\}\right\}$, and $\Lambda_{f}=\left\{z \mid z\right.$ is an limit element of $\left.\left\{f\left(x_{d}\right)\right\}\right\}$. Then $f$ is continuous at $x \Leftrightarrow\left(\forall\left\{x_{d} \mid d \in D\right\}\right)\left(x_{d x} \rightarrow x \Rightarrow f\left(x_{d}\right) \rightarrow f(x)\right)$. 
Proof: Let $f$ be continuous and $x_{d} \rightarrow x$. By Lemma 13(ii), $f(x) \in \Lambda_{f}$. We must prove that $\left(\forall t \in \Lambda_{f}\right)(f(x) \leqslant t)$. Let $t \in \Lambda_{f}$, and $g \in \eta_{2}(t) \cap G_{2}$. $(\exists q \in D)(t \leqslant g \& p \geqslant q) \Rightarrow f\left(x_{p}\right) \leqslant g$. Thus, $p \geqslant q \Rightarrow x_{p} \leqslant f^{+}\left(f\left(x_{p}\right)\right) \leqslant f^{+}(g)$. Hence, $f^{+}(g) \in \Lambda \cap \eta_{1}(x)$, and $x \leqslant f^{+}(g) \Rightarrow f^{+}(g) \in \eta_{1}(x)$. By Lemma 13(i), $\eta_{2}(t) \subseteq \eta_{2}(f(x))$. By Theorem 5, $f(x) \leqslant t$. Conversely, suppose that $\left(\forall\left\{x_{d} \mid d \in D\right\}\right)\left(x_{d} \rightarrow x \Rightarrow f\left(x_{d}\right) \rightarrow f(x)\right)$. From Lemma 18(i), Theorem 4, and Corollary 10.1, we have $\eta_{1}(x) \rightarrow x, \Lambda=[x), f(x)=\bigwedge_{w \in \eta_{1}(x)} f(w), \eta_{2}(f(x)) \subseteq \Lambda_{f}$, and $f_{*}\left(\eta_{1}(x)\right) \rightarrow f(x)$. Now, $g \in \eta_{2}(f(x)) \Rightarrow\left(\exists q \in \eta_{1}(x)\right) \quad\left(f(q) \leqslant g \Rightarrow q \leqslant f^{+}(f(q))\right.$ $\left.\leqslant f^{+}(g)\right)$. Thus, $f^{+}(g) \in \eta_{1}(x)$.

It follows inmediately from Theorem 15 that if $\left(X_{1}, T_{1}\right)$ and $\left(X_{2}, T_{2}\right)$ are $T_{1}$-topological spaces then a function $f: X_{1} \rightarrow X_{2}$ is continuous if and only if $\left(\forall B \in \mathcal{P}\left(X_{1}\right)\right)\left(\forall\left\{B_{d} \mid d \in D\right\}\right.$ a net in $\left.\mathcal{P}\left(X_{1}\right)\right)\left(B_{d} \rightarrow B \Rightarrow f_{*}\left(B_{d}\right) \rightarrow f_{*}(B)\right)$. Thus, we have shown that in statements concerning continuity and the convergence of nets in $T_{1}$-topological spaces, points can be replaced by sets, neighbourhoods of points by neighbourhoods of sets, and the point function $f$ by the lattice function $f_{*}$.

\section{CONVERGENCE OF FILTERS IN NEIGHBOURHOOD LATTICES}

We begin our development of filter convergence by observing that if $F$ is a filter in a lattice $L$, then $(F, \leqslant)$ is a directed set. Thus, $F$ is a net in $L$. If $F$ is a filter in a lattice $L$, we will write $F \rightarrow x$ to indicale that the filter $F$ converges to $x$ as a net. Further, we will let $\Lambda_{F}$ represent the set of limit elements of the filter $F$.

Definition 16: If $(L, \eta)$ is a conditionally complete $T_{1}$-lattice, and $x \in L$, then we will say that $F$ converges to $X$ as a filter, written $F \stackrel{f}{\rightarrow} x$, if $\eta(x) \subseteq F$.

If we attempt to replace point by set in the definition of filter convergence, then, even in a Hausdorff topological space, we are faced with non-uniqueness of limits. For example, if $F \stackrel{f}{\rightarrow} A$, and $A \subseteq B$, then $F \stackrel{f}{\rightarrow} B$. The remainder of this section is devoted to examining the relationship between net and filter convergence.

Lemma 17. Let $(L, \eta)$ be a neighbourhood lattice, $F$ be a filter in $L$, and $\{x \mid$ $x \in F\}$ be the net corresponding to $F$. Then

(i) $t \in \Lambda_{F} \Leftrightarrow(\forall g \in \eta(t) \cap G)(\exists q \in F)(q \leqslant g)$;

(ii) $\Lambda_{F}=\{t \mid F \stackrel{f}{\rightarrow}, t\}$;

(iii) $F \stackrel{f}{\rightarrow} x \Leftrightarrow F \cap[x) \stackrel{f}{\rightarrow} x$;

(iv) $F \rightarrow x \Rightarrow F \cap[x) \rightarrow x$;

(v) $F \subseteq \Lambda_{F}$;

(vi) $\Lambda_{F}=\emptyset \Leftrightarrow F=\emptyset$. 
Lemma 17(ii) establishes that if $F$ is a filter, then the set of limit elements of the net $F$ is exactly the set of elements to which $F$ converges as a filter. Further, it follows from Lemma 17 (ii), that if $(L, \eta)$ is a conditionally complete $T_{1}$-lattice, then $F \rightarrow x \Rightarrow F \stackrel{f}{\rightarrow} x$. Lemma 17(iii) shows that if $F$ is a filter, then the filter convergence of $F$ is determined by the part of $F$ that is contained in $[x$ ). Finally, Lemma 17(vi) proves that any filter $F$ other than the null filter $F=\emptyset$ has a non-void set of limit elements.

Lemma 18 was used in the proof of Theorem 15. It is also a useful first step towards determining set theoretic bounds for convergent filters.

LEMMA 18. Let $(L, \eta)$ be a conditionally complete $T_{1}$-lattice and $F$ be a filter in L. Then

$$
\begin{aligned}
\text { (i) } & \eta(x) \rightarrow x, \\
\text { (ii) } & {[x) \rightarrow x, } \\
\text { (iii) } & \left\{x_{d} \mid d \in D\right\} \rightarrow x \Rightarrow \Lambda \stackrel{f}{\rightarrow} x .
\end{aligned}
$$

Proof: (i) $t \in \Lambda_{\eta(x)} \Leftrightarrow \eta(x) \stackrel{f}{\rightarrow} t \Leftrightarrow \eta(t) \subseteq \eta(x) \Leftrightarrow x \leqslant t \Leftrightarrow t \in[x)$. (ii) $\Lambda_{[x]}=\{t: \eta(t) \subseteq[x]\}$. Thus, $x \in \Lambda_{[x]}$, and $[x] \subseteq \Lambda_{[x]}$. Now, $t \in \Lambda_{[x]} \Rightarrow$ $(\forall g \in \eta(t))(\exists q \in[x))(q \leqslant g \Rightarrow g \in \eta(x)) \Rightarrow \eta(t) \subseteq \eta(x) \Rightarrow x \leqslant t \Rightarrow t \in[x)$. Hence $\Lambda_{[x]}=[x)$.

Theorem 19 gives set theoretic bounds for convergent filters in conditionally complete $T_{1}$-lattices.

Theorem 19. Let $(L, \eta)$ be a conditionally complete $T_{1}$-lattice, $x \in L$, and $F$ be a filter in $L$. Then $F \rightarrow x \Leftrightarrow \eta(x) \subseteq F \subseteq[x)$.

Proof: $F \rightarrow x \Rightarrow \Lambda_{F}=[x) \Rightarrow \eta(x) \subseteq F \subseteq \Lambda_{F}=[x)$. Conversely, $\eta(x) \subseteq F \subseteq[x) \Rightarrow x \in \Lambda_{F} \Rightarrow[x) \subseteq \Lambda_{F}$. Now, $t \in \Lambda_{F} \Rightarrow \eta(t) \subseteq F \subseteq[x) \Rightarrow$ $(\forall g \in G \cap \eta(t))(x \leqslant g \Rightarrow \eta(t) \subseteq \eta(x)) \Rightarrow x \leqslant t \Rightarrow t \in[x)$. Hence $\Lambda_{F}=[x)$.

CoRollary 19.1. Let $(L, \eta)$ be a conditionally complete $T_{1}$-lattice, $x \in L$, and $F$ be a filter in $L$. Then:

(i) $F \rightarrow x \Rightarrow x=\bigwedge_{x \in F} z$;

(ii) let $F$ be a proper filter; then $F \rightarrow x \Rightarrow x \neq \emptyset$;

(iii) $F \stackrel{f}{\rightarrow} x \Leftrightarrow F \cap[x) \rightarrow x$;

(iv) let $x=\bigwedge_{z \in F} z \in L$; then $F \rightarrow x \Leftrightarrow F \stackrel{f}{\rightarrow} x$.

PROOF: (i) $F \rightarrow x \Rightarrow \eta(x) \subseteq F \subseteq[x) \Rightarrow \bigwedge_{z \in F} z=x$.

(ii) $F \rightarrow x \& x=\emptyset \Rightarrow F=L$. A contradiction. 
(iii) $F \stackrel{f}{\rightarrow} x \Rightarrow \eta(x) \subseteq F$. But $\eta(x) \subseteq[x) \Rightarrow \eta(x) \subseteq F \cap[x) \subseteq[x) \Rightarrow F \cap[x) \rightarrow x$. Conversely, $F \cap[x) \rightarrow x \Rightarrow \eta(x) \subseteq F \cap[x) \subseteq[x] \Rightarrow F \cap[x) \stackrel{f}{\rightarrow} x \Rightarrow F \stackrel{f}{\rightarrow} x$.

(iv) $F \rightarrow x \Rightarrow \Lambda_{F}=[x) \Rightarrow F \stackrel{f}{\rightarrow} x$. Conversely, $F \stackrel{f}{\rightarrow} x \Rightarrow \eta(x) \subseteq F \Rightarrow[x) \subseteq \Lambda_{F}$. But, $\eta(y) \subseteq F \Rightarrow x \leqslant \bigwedge_{z \in \eta(y)} z=y \Rightarrow \Lambda_{F} \subseteq[x)$.

COROLlaRY 19.2. Let $(X, T)$ be a $T_{1}$-topological space, $(\mathcal{P}(X), \eta)$ be the induced neighbourhood lattice, $F$ be a filter in $\mathcal{P}(X), x \in X$, and $\{x\}=\bigwedge_{x \in F} z$. $F \rightarrow\{x\} \Leftrightarrow F \stackrel{f}{\rightarrow}\{x\}$.

Corollary 19.2 shows that if the infimum of the filter $F$ is a singleton set, then the net convergence of $F$ is necessary and sufficient for the filter convergence of $F$. Further, Example 23 will show that if $\emptyset=\bigwedge_{x \in F} z$, then $F \stackrel{f}{\rightarrow} x$ need not imply $F \rightarrow x$. This example also disproves the converse of Corollary 19.1(i).

We will now prove that in a topological space, a net of sets converges to $x$ if and only if the corresponding Fréchet filter converges as a net to $x$.

Definition 20: Let $D$ be a directed set, $\left\{x_{d} \mid d \in D\right\}$ be a net in a topological space $(X, T)$, and $(\forall k \in D)\left(A_{k}=\left\{x_{d} \mid d \geqslant k\right\}\right)$. If we let $F_{r}=\left\{B \mid(\exists q \in D)\left(A_{q} \subseteq B\right)\right\}$, then $F_{r}$ is the Fréchet filter or elementary filter associated with $\left\{x_{d} \mid d \in D\right\}$.

TheOREM 21. Let $\left\{x_{d} \mid d \in D\right\}$ be a net in a topological space $(X, T)$ and $\Lambda$ be the set of limit elements of $\left\{x_{d} \mid d \in D\right\}$. Then

(i) $\Lambda=\Lambda_{F_{r}}$,

(ii) $\left\{x_{d} \mid d \in D\right\} \rightarrow x \Leftrightarrow F_{r} \rightarrow x$.

Proof: (i) $t \in \Lambda_{F} \Leftrightarrow(\forall g \in G \cap \eta(t))\left(\exists q \in F_{r}\right)(q \leqslant g) \Leftrightarrow(\forall g \in G \cap \eta(t))$ $\left(\exists d_{0} \in D\right)\left(A_{d_{0}} \subseteq g\right)$. Now, $d>d_{0} \Rightarrow A_{d} \subseteq A_{d_{0}}$. Hence, $(\forall g \in G \cap \eta(t))\left(\exists d_{0} \in D\right.$ $\left.\& d>d_{0}\right)\left(A_{d} \subseteq g\right)$. Thus $\Lambda_{F_{r}} \subseteq \Lambda$. Conversely, $t \in \Lambda \Rightarrow(\forall g \in G \cap \eta(t))\left(\exists d_{0} \in D\right.$ $\left.\& d>d_{0}\right)\left(x_{d} \subseteq g\right) \Rightarrow A_{d_{0}} \subseteq g \Rightarrow \eta(t) \subseteq F_{r} \Rightarrow t \in \Lambda_{F_{r}}$.

(ii) follows immediately from (i).

Corollary 21.1. Let $\left\{x_{d} \mid d \in D\right\}$ be a net in a topological space $(X, T)$ and $\Lambda$ be the set of accumulation elements of $\left\{x_{d} \mid d \in D\right\}$. Then

(i) $\left\{x_{d} \mid d \in D\right\} \rightarrow y \Leftrightarrow F_{r} \rightarrow y \Leftrightarrow(\forall x \in y)\left(F_{r} \stackrel{f}{\rightarrow}\{x\}\right)$;

(ii) if $(X, T)$ is a Hausdorff space, then $\left\{x_{d} \mid d \in D\right\} \rightarrow\{x\} \Leftrightarrow F_{r} \rightarrow\{x\} \Leftrightarrow$ $F_{\mathrm{r}} \stackrel{f}{\rightarrow}\{x\}$.

Proof: (i) This follows from Theorem 21 and the usual topological result that a net converges to $x \in X$ if and only if the corresponding Fréchet filter converges to $x$ 
as a filter.

(ii) This follows from (i) and the uniqueness of limits in a Hausdorff space.

Lemma 22. Let $(L, \eta)$ be a complete $T_{1}$-lattice and $F$ be a filter in $L$. Then $F \stackrel{f}{\rightarrow} x \& F \stackrel{f}{\rightarrow} y \Rightarrow w=\bigwedge_{z \in F} z \leqslant x \wedge y$.

PROOF: $F \stackrel{f}{\rightarrow} x \& F \stackrel{f}{\rightarrow} y \Rightarrow \eta(x) \subseteq F \& \eta(y) \subseteq F \Rightarrow \bigwedge_{z \in F} z \leqslant x \& \bigwedge_{z \in F} z \leqslant y \Rightarrow$ $w=\bigwedge_{z \in F} z \leqslant x \wedge y$

In particular, if we apply Lemma 22 to a filter $F$ in a $T_{1}$-topological space, then $F \stackrel{f}{\rightarrow}\{x\}, F \stackrel{f}{\rightarrow}\{y\}$ and $x \neq y$ implies $w=\bigcap_{z \in F} z=\emptyset$. Thus $w=\bigcap_{z \in F} z \neq \emptyset$ implies that if $F$ converges as a filter to a singleton set, then the limit is unique.

Example 23. Let $\mathbf{R}^{+}=\{x \mid x>0\}$ and define $\left(\forall \delta \in \mathbf{R}^{+}\right)\left(A_{\delta}=(0,1 / \delta)\right)$. Further, let $\Lambda$ be the set of limit elements of the net $\left\{A_{\delta} \mid \delta \in \mathbf{R}^{+}\right\}$and let $F_{r}$ be the filter generated by $\left\{A_{\delta} \mid \delta \in \mathbf{R}^{+}\right\}$, that is, $F_{r}=\left\{w \mid\left(\exists \delta \in \mathbf{R}^{+}\right)\left(A_{\delta} \subseteq w\right)\right\}$.

(i) Since $\bigcap_{x \in \Lambda} z=\emptyset \notin \Lambda,\left\{A_{\delta}\right\}$ does not converge as a net. By Theorem $21, F_{r}$ does not converge as a net.

(ii) Clearly, $\eta(\{0\}) \subseteq F_{r}$. If $x$ is any other singleton set, then there is an $A_{\delta}$ such that $A_{\delta} \notin \eta(x)$. Hence, $x$ is a singleton set and $\eta(x) \subseteq F_{r} \Leftrightarrow x=\{0\}$. Thus, $F_{r} \stackrel{f}{\rightarrow}\{0\}$. By Lemma 17(iii), $F_{r} \cap[\{0\}) \stackrel{f}{\rightarrow}\{0\}$.

(iii) Although $F_{r} \stackrel{f}{\rightarrow}\{0\},\{0\} \notin F_{r}, F_{r}$ is not a subset of $[\{0\})$, and neither $F_{r}$ nor $F_{r} \cap[\{0\})$ are principal filters. Further, $\bigcap_{z \in F_{r}} z=0$, but $\bigwedge_{z \in F_{r} \cap(\{0\})} z=\{0\} \notin F_{r} \cap[\{0\})$. Lastly, $\eta(\{0\}) \subseteq F_{r} \cap[\{0\}) \subseteq[\{0\})$. Hence by Theorem 21, $F_{r} \cap[\{0\}) \rightarrow\{0\}$.

In considering the convergence properties of maximal filters we make use of the fact that.if $F$ is a proper maximal filter in a lattice $L$ with $\emptyset$ and $\bigwedge_{x \in F} z=x \in L$, then either $F$ is a principal filter, that is, $F=[x]$, or $x=\emptyset$.

Theorem 24. Let $(L, \eta)$ be a complete $T_{1}$-lattice and $F$ be a filter in $L$. Then

(i) $F$ is a proper filter $\Leftrightarrow \Lambda_{F} \neq L$;

(ii) let $F$ be a proper maximal filter, then $F \rightarrow x \Rightarrow F=\Lambda_{F}$;

(iii) let $F$ be a proper maximal filter and $\bigwedge_{z \in F} z=x$, then $F \rightarrow x \Leftrightarrow x \neq \emptyset$.

Proof: (i) $\Lambda_{F}=L \Rightarrow \emptyset \in \Lambda_{F} \Rightarrow \eta(\emptyset) \subseteq F \Rightarrow F=L$. Conversely, $F=L$ and $F \subseteq \Lambda_{F} \Rightarrow \Lambda_{F}=L$.

(ii) $F \subseteq \Lambda_{F}$ and $\Lambda_{F}$ a filter $\Rightarrow$ either $F=\Lambda_{F}$ or $\Lambda_{F}=L$. But by (i) $\Lambda_{F}=L \Rightarrow F=$ $L$. A contradiction. 
(iii) $\bigwedge_{z \in F} z=x \neq \emptyset \Rightarrow F=[x)=\Lambda_{F}$. By Lemma $17($ ii),$F \rightarrow x$. The converse follows from Corollary 19.1(i).

Definition 25: Let $L$ be a lattice, and $A \subseteq L$. We will define the filter generated by $A$, denoted $\langle A\rangle$, by $\langle A\rangle=\cap\{F \mid F$ is a filter $\& A \subseteq F\}$.

We will now discuss the relationship between the convergence of a filter $F$ and the convergence of the filter generated by $\Lambda_{F}$. Particular attention will be paid to those filters which converge to a singleton set in $\mathcal{P}(X)$. In Lemma 26, we will include the lattice theoretic properties of filters that will be needed.

Lemma 26. Let $L$ be a lattice, and $A \subseteq L$. Then
(i) $\langle A\rangle=\bigvee_{a \in A}[a)$;
(ii) if $\bigwedge_{a \in A} a$ exists in $A$, then $\langle A\rangle=\left[\bigwedge_{a \in A} a\right)$;
(iii) $\bigvee_{\alpha \in I}\left(x_{\alpha}\right) \subseteq\left[\bigwedge_{\alpha \in I} x_{\alpha}\right)$;
(iv) $\left\langle\bigcup_{a \in A}[a)\right\rangle=\bigvee_{a \in A}[a)$.

LeMma 27. Let $(L, \eta)$ be a conditionally complete $T_{1}$-lattice, $F$ a filter in $L$ and $x \in L$. Then $\left\langle\Lambda_{F}\right\rangle \rightarrow x \Rightarrow \forall a \in \Lambda_{F}, x \leqslant a$, and $\bigwedge_{a \in \Lambda_{F}} a=a_{0}$ exists in $L$.

PRoof: $\left\langle\Lambda_{F}\right\rangle \rightarrow x \Leftrightarrow \Lambda_{\left\langle\Lambda_{F}\right\rangle}=[x)=\left\{t: \eta(t) \subseteq\left\langle\Lambda_{F}\right\rangle\right\}$. Now, $\Lambda_{F} \subseteq\left\langle\Lambda_{F}\right\rangle \subseteq$ $\Lambda_{\left\langle\Lambda_{F}\right\rangle}=[x) \Rightarrow \forall a \in \Lambda_{F}, x \leqslant a$. Hence $\bigwedge_{a \in \Lambda_{F}} a=a_{0}$ exists in $L$.

Lemma 28. Let $(X, T)$ be a $T_{1}$-topological space, $(\mathcal{P}(X), \eta)$ be the induced neighbourhood lattice and $F$ be a filter in $\mathcal{P}(X) .\left\langle\Lambda_{F}\right\rangle \rightarrow\{x\} \Rightarrow \Lambda_{F}$ is a filter, $\Lambda_{F} \rightarrow\{x\}$ and $F \rightarrow\{x\}$.

Proof: $\left\langle\Lambda_{F}\right\rangle \rightarrow\{x\} \Rightarrow \forall a \in \Lambda_{F},\{x\} \subseteq a \Rightarrow\left(\forall a \in \Lambda_{F}\right)$
$\left(\eta(a) \subseteq \eta(\{x\}) \Rightarrow \bigcap_{a \in \Lambda_{F}} \eta(a) \subseteq \eta(\{x\})\right) \Rightarrow \eta\left(\bigcup_{a \in \Lambda_{F}} a\right) \subseteq \eta(\{x\}) \Rightarrow \eta\left(\Lambda_{F}\right) \subseteq \eta(\{x\}) \Rightarrow$
$\{x\} \subseteq \Lambda_{F} \Rightarrow\left[\{x\} \subseteq \subseteq \Lambda_{F}\right.$. But, by Lemma 27 , we have $\Lambda_{F} \subseteq\{\{x\})$. Hence, $\Lambda_{F}=[\{x\})=\left\langle\Lambda_{F}\right\rangle=\Lambda_{\left\langle\Lambda_{F}\right\rangle}$. Thus, $\Lambda_{F}$ is a principal filter, and $F \rightarrow\{x\}$.

Theorem 29. Let $(X, T)$ be a $T_{1}$-topological space, $(\mathcal{P}(X), \eta)$ be the induced neighbourhood lattice, and $F$ be a filter in $\mathcal{P}(X)$. Then

(i) $F \rightarrow\{x\} \Leftrightarrow\left\langle\Lambda_{F}\right\rangle \rightarrow\{x\}$;

(ii) $F \rightarrow\{x\} \Leftrightarrow \Lambda_{F}$ is a filter and $\Lambda_{F} \rightarrow\{x\}$. 
Proof: (i) $F \rightarrow\{x\} \Rightarrow \Lambda_{F}=[\{x\}) \Rightarrow\left\langle\Lambda_{F}\right\rangle=[\{x\}) \Rightarrow\left\langle\Lambda_{F}\right\rangle \rightarrow x$. Lemma 28 proves the converse.

(ii) If $\Lambda_{F}$ is a filter, then $\Lambda_{F}=\left\langle\Lambda_{F}\right\rangle$, and the result follows from (i).

We will now show how topologies can be 'lifted' from $X$ to $P(X)$ and then how this 'lifted' topology can be used to prove that continuous functions preserve the limits of convergent filters.

LEMMA 30. Let $(L, \eta)$ be a pre-neighbourhood lattice with greatest element 1 and let $G$ be the set of open elements of $L$. If $\beta=\{(g] \mid g \in G\}$, then $\beta$ is the base for a topology $T$ on $L$. Further, $A \in T \Leftrightarrow(\exists B \in \mathcal{P}(G))\left(A=\bigcup_{b \in B}(b]\right)$.

Proof: $L=(1] \in \beta .\left(g_{1}\right],\left(g_{2}\right] \in \beta \Rightarrow\left(g_{1}\right] \cap\left(g_{2}\right]=\left(g_{1} \wedge g_{2}\right) \in \beta$.

Theorem 31. Let $\left(L_{1}, \eta_{1}\right)$ and $\left(L_{2}, \eta_{2}\right)$ be pre-neighbourhood lattices, each with a greatest element, and, for $i=1,2$, let $T_{i}$ be the topology on $L_{i}$ with base $\beta_{i}=\left\{(g]: g \in G_{i}\right\}$ and let $\left(P\left(L_{i}\right), \tilde{\eta}_{i}\right)$ be the induced neighbourhood lattices. If $f:\left(L_{1}, \eta_{1}\right) \rightarrow\left(L_{2}, \eta_{2}\right)$ is $(\eta)$ continuous and residuated, then $f_{*}:\left(P\left(L_{1}\right), \tilde{\eta}_{1}\right) \rightarrow$ $\left(P\left(L_{2}\right), \tilde{\eta}_{2}\right)$ is $(\tilde{\eta})$ continuous. Thus, $f: L_{1} \rightarrow L_{2}$ is topologically continuous.

Proof: $A \in T_{2} \Rightarrow(\exists B \in \mathcal{P}(G))\left(A=\bigcup_{b \in B}(b]\right)$. But, if $f: L_{1} \rightarrow L_{2}$ is a residuated mapping, then $\left(\forall x \in L_{2}\right)\left(\left(f^{+}(x)\right]=f^{*}((x])\right)$. Thus, $f^{*}(A)=f^{*}\left(\bigcup_{b \in B}(b]\right)=$ $\bigcup_{b \in B} f^{*}((b])=\bigcup_{b \in B}\left(f^{+}(b)\right]$. Now $f$ continuous and $b \in G_{2} \Rightarrow f^{+}(b) \in G_{1}$. Thus $f^{*}(A) \in T_{1}$.

Example 32. Let $\left(X, T_{1}\right)$ and $\left(Y, T_{2}\right)$ be topological spaces, $f: X \rightarrow Y$ be a continuous function and $\left(\mathcal{P}(X), \eta_{1}\right),\left(\mathcal{P}(Y), \eta_{2}\right)$ be the induced neighbourhood lattices. We can apply Theorem 31 to $f_{*}:\left(\mathcal{P}(X), \eta_{1}\right) \rightarrow\left(\mathcal{P}(Y), \eta_{2}\right)$, which is $(\eta)$ continuous and residuated. Thus, we have $f_{* *}:\left\{\left(\mathcal{P}(\mathcal{P}(X)), \tilde{\eta}_{1}\right) \rightarrow\left(\mathcal{P}(\mathcal{P}(Y)), \tilde{\eta}_{2}\right)\right.$ is $(\tilde{\eta})$ continuous and residuated. But $\tilde{\eta}_{1}$ and $\tilde{\eta}_{2}$ determine topologies on $\mathcal{P}(X)$ and $\mathcal{P}(Y)$ respectively, and $f_{*}$ is topologically continuous since $\left(f_{*}\right)_{*}$ is $(\bar{\eta})$ continuous. We will say that the $\tilde{\eta}$ neighbourhood system 'lifts' the topology from $X$ to $\mathcal{P}(X)$ and the neighbourhood system from $\mathcal{P}(X)$ to $\mathcal{P}(\mathcal{P}(X))$.

In Theorem 33 we will prove that, in the appropriate neighbourhood systems, the continuous image of a (net) convergent filter is a (net) convergent filter.

Theorem 33. Let $\left(X, T_{1}\right)$ and $\left(Y, T_{2}\right)$ be $T_{1}$-topological spaces, $f: X \rightarrow Y$ be a continuous function and let $\mathcal{P}(X)$ and $\mathcal{P}(Y)$ have the topologies 'lifted' respectively from $X$ and $Y$. Then

(i) $F \rightarrow x \Rightarrow f_{* *}(F) \rightarrow\left\{f_{*}(x)\right\}$; 
(ii) in particular, $F \rightarrow\{x\} \Rightarrow f_{* *}(F) \rightarrow\{\{f(x)\}\}$; When $F=\eta(x)$, we have $\eta(x) \rightarrow\{x\} \Rightarrow f_{* *}(\eta(x)) \rightarrow\{\{f(x)\}\}$.

Proof: $f$ is continuous $\Leftrightarrow f_{*}$ is continuous $\Leftrightarrow f_{* *}$ is continuous. Now apply Theorem 15.

It is clear that there is no direct filter convergence analogue of Theorem 33, since if $F$ is a filter in $\mathcal{P}(X)$ and $f: X \rightarrow Y$ is a function, $f_{* *}(F)$ is a net, and although a filter base, need not be a filter.

We will now show that if $L$ is a conditionally complete neighbourhood lattice we can define a neighbourhood system on $\mathcal{F}(L)$ in such a way that $\left(\left\{x_{d} \mid d \in D\right\} \rightarrow x\right) \Leftrightarrow$ $\left(\eta\left(x_{d}\right) \rightarrow \eta(x)\right)$. We begin by observing that if $\left\{x_{d}\right\}$ is a net in $L$, then $\left\{\eta\left(x_{d}\right)\right\}$ is a net of filters in $\mathcal{F}(L)$. Further, $(\mathcal{F}(L), \vee, \wedge)$ is a lattice if we define $F_{1} \leqslant F_{2}$ if and only if $F_{2} \subseteq F_{1}, F_{1} \vee F_{2}=F_{1} \cap F_{2}$, and $F_{1} \wedge F_{2}=\cap\left\{F \mid F \in \mathcal{F}(L) \& F \leqslant F_{1}, F \leqq F_{2}\right\}$, that is $\leqslant$ is the "usual" ordering of the lattice of filters of a poset.

LEMMA 34. Let $(L, \eta)$ be a conditionally complete $T_{1}$ neighbourhood lattice, $G$ be th set of open elements of $L$, and let $\left\{x_{d}\right\}$ be a net in $L$. Then

(i) $\left(\mathcal{F}(L), \eta_{1}\right)$ is a neighbourhood lattice if we define $F \in \mathcal{F}(L)$ to be open $\Leftrightarrow(\exists g \in G \& F=[g))$.

(ii) $\eta: L \rightarrow \mathcal{F}(L)$ is a continuous $1-1$ open residuated function.

\section{ProOF:}

(i) This follows from Lemma 26.

(ii) This follows from the definition of $\eta^{+}: \mathcal{F}(L) \rightarrow L$ by $\eta^{+}(F)=\bigwedge_{f \in F} f^{0}$.

Theorem 35. Let $(L, \eta)$ be a conditionally complete $T_{1}$ neighbourhood lattice, $\left\{x_{d}\right\}$ be a net in $L,\left(\mathcal{F}(L), \eta_{1}\right)$ be the corresponding neighbourhood lat tice of filters of $L$, and $G_{1}$ be the set of open elements of $\mathcal{F}(L)$.

$$
x_{d} \rightarrow x \Leftrightarrow \eta\left(x_{d}\right) \rightarrow \eta(x) .
$$

Proof: By Lemma 34 and Theorem 16, $x_{d} \rightarrow x \Rightarrow \eta\left(x_{d}\right) \rightarrow \eta(x)$. Conversely, to prove $\eta\left(x_{d}\right) \rightarrow \eta(x) \Rightarrow x_{d} \rightarrow x$, we must show that $\Lambda=[x) . g \in \eta(x) \cap G \Rightarrow$ $[g) \subseteq \eta(x) \Rightarrow \eta(x) \leqslant[g) \Rightarrow[g) \in \eta_{1}(\eta(x)) \cap G_{1} \Rightarrow(\exists 1 \in D)(\forall p \in D)\left(p \geqslant q \Rightarrow \eta\left(x_{p}\right)\right.$ $\leqslant(g)=\eta(g)) \Rightarrow(\exists q \in D)(\forall p \in D)\left(p \geqslant q \Rightarrow x_{p} \leq g\right)$. Thus $[x) \subseteq \Lambda$. Let $a \in \Lambda$. $(\forall g \in \eta(a) \cap G)(\exists q \in D)\left(p \geqslant q \Rightarrow x_{p} \leqslant g\right)$. Now, $\tilde{g} \in \eta_{1}(\eta(a)) \cap g_{1} \Rightarrow \eta(a) \leqslant \tilde{g}=$ $\left[g_{1}\right)=\eta\left(g_{1}\right) \Rightarrow a \leqslant g_{1} \Rightarrow(\exists q \in D)\left(p \geqslant q \Rightarrow x_{p} \leqslant g_{1}\right) \Rightarrow(\exists q \in D)\left(p \geqslant q \Rightarrow \eta\left(x_{p}\right)\right.$ $\left.\leqslant \eta\left(g_{1}\right)=\widetilde{g} \Rightarrow \eta(a) \in \Lambda_{\eta}\right)$. Hence, $\eta(x) \leqslant \eta(a)$, and by Theorem $5, x \leqslant a$. 
In Theorem 21, it was proved that in a topological space a net of sets converges to $\boldsymbol{x}$ if and only if the corresponding Fréchet filter converges as a net to $x$. However, the construction of the Fréchet filter is dependent on the lattice structure of $\mathcal{P}(X)$. We will conclude this paper by constructing a filter generated by the neighbourhood systems of the elements of a net in any neighbourhood lattice which will replace the Fréchet filter in the lattice analogue of Theorem 21.

Lemma 36. Let $(L, \eta)$ be a neighbourhood lattice and let $\left\{x_{d}\right\}$ be a net in $L$. Then

(i) $x \in \Lambda \Leftrightarrow(\forall g \in G \cap \eta(x))\left(\exists d_{0} \in D\right)(\forall d \in D)\left(d \geqslant d_{0} \Rightarrow g \in \eta\left(x_{d}\right)\right)$,

(ii) $x \in \Lambda \Leftrightarrow\left(\exists d_{0} \in D\right)(\forall d \in D)\left(d \geqslant d_{0} \Rightarrow \eta(x) \subseteq \eta\left(x_{d}\right)\right)$,

(ii) $x \in \Lambda \Leftrightarrow\left(\exists d_{0} \in D\right)\left(\eta(x) \subseteq \bigcap_{k \geqslant d_{0}} \eta\left(x_{k}\right)\right)$.

If $(L, \eta)$ is a neighbourhood lattice and $\left\{x_{d}\right\}$ a net in $L$, we will define $(\forall d \in D)$ $\left(F_{d}=\bigcap_{k \geqslant d} \eta\left(x_{k}\right)=\eta\left(\bigvee_{k \geqslant d} x_{k}\right)\right)$. We note that $(\forall d \in D)\left(F_{d}\right.$ is a filter in $\left.L\right)$, and that if we let $\mathcal{F}=\bigcup_{d \in D} F_{d}$, then $\mathcal{F}$ is a filter in $L$.

TheOREM 37. Let $(L, \eta)$ be a neighbourhood lattice, let $\left\{x_{d}\right\}$ be a net in $L$, and let $\mathcal{F}=\bigcup_{d \in D} F_{d}$. Then

(i) $\Lambda=\Lambda_{\mathcal{F}}$ and

(ii) $\left\{x_{d} \mid d \in D\right\} \rightarrow x \Leftrightarrow \mathcal{F} \rightarrow x$.

\section{Proof:}

(i) By Lemma 36(iii), $\Lambda \subseteq \Lambda_{\mathcal{F}}$. Now, $y \in \Lambda_{\mathcal{F}} \Rightarrow \eta(y) \subseteq \mathcal{F}$. Thus, $g \in \eta(y) \cap$

$$
\begin{aligned}
& G \Rightarrow g \in \mathcal{F}=\bigcup_{d \in D} F_{d} \Rightarrow\left(\exists d_{g} \in d\right)\left(g \in F_{d_{g}}\right) \Rightarrow\left(\exists d_{g} \in D\right)\left(g \in \bigcap_{k \geqslant d_{g}} \eta\left(x_{k}\right)\right) \\
& \Rightarrow\left(\exists d_{g} \in D\right)\left(k \geqslant d_{g}\right) \Rightarrow g \in \eta\left(x_{k}\right) \Rightarrow \in \Lambda .
\end{aligned}
$$

(ii) This follows from (i).

\section{REFERENCES}

[1] C. Berge, Topological spaces (Oliver and Boyd, Edinburgh, 1963).

[2] G. Birkhoff, Lattice theory (A.M.S., Providence, 1967).

[3] T.S. Blythe and M.F. Janowitz, Residuation theory (Pergammon Press, Oxford, 1972).

[4] N. Bourbaki, General topology, Part 1 (Addison-Wesley, Reading, Mass., 1966).

[5] E. Cech, Topological spaces, Vols I and II (Interscience Publishers, London, N.Y., Sydney, 1966).

[6] G. Choquet, 'Convergences', Grenoble Universite Annales 23 (1947), 57-112. 
[7] W.W. Comfort and S. Negrepontis, The theory of ultrafilters (Springer-Verlag, Berlin, Heidelberg, 1974).

[8] J. Dugundji, Topology (Allyn and Bacon, Inc., Boston, 1966).

[0] S. Eilenberg, 'Ordered topological spaces', Amer. J. Math. 63 (1941), 39-45.

[10] S.A. Gaal, Point oet topology (Academic Press, New York, 1964).

[11] G. Gratzer, Lattice theory: first concepts and distributive lattices (Freeman, San Francisco, 1971).

[12] F. Hausdorf, Mengenlehre (Berlin-Leipzig, 1927).

[13] J.L. Kelley, General topology (D. Van Nostrand Co., Inc., Princeton, New Jersey, 1955).

[14] K. Kuratowski, Introduction to set theory and topology (Addison-Wesley, Reading, Mass,, 1962).

[15] C. Kuratowski, 'Sur la notion de limite topologique d'ensembles', Polski Towarzystwo Matematyczne Rocznik 21 (1949), 219-225.

[16] C. Kuratowski, 'Sur les familles monotones d'ensembles fermes et leurs applications à la theorie des espaces commexes', Fund. Math. 30 (1938), 17-33.

[17] S. Mrowa, 'On the convergence of nets of sets', Fund. Math. 45 (1958), 237-246.

[18] L. Nachbin, Topology and order (D. Van Nostrand Co, Inc., Princeton, New Jersey, 1965).

[19] Z.F. Prahs, 'Conceruing topological convergence of sets', Czechoslovak Math. J. 10:(1960), 168-179.

[20] F.P. Prokop, 'Neighbourhood lattices-a poset approach to topological spaces', Bull. Austral. Math. Soc. 39 (1989), 31-48.

[21] F.P. Prokop, Ph.D. thesis (Univ. of Wollongong, Wollongong, N.S.W., 1980).

[22] R. Sikorsky, Boolean algebras (Springer-Verlag, Berlin, Heidelberg, New. York, 1964).

Department of Mathematics

The University of Wollongong

Wollongong, N.S.W.

Australia 